\title{
A Neumann System of the Third Order Differential Operator Associated with the Boussinesq Equation
}

\author{
Jianli Cao*, Jingfang Han \\ College of Science, Henan University of Technology, Zhengzhou, China \\ Email: *cao_j_1@163:com
}

How to cite this paper: Cao, J.L. and Han, J.F. (2020) A Neumann System of the Third Order Differential Operator Associated with the Boussinesq Equation. Journal of Applied Mathematics and Physics, 8, 2861-2868. https://doi.org/10.4236/jamp.2020.812211

Received: October 19, 2020

Accepted: December 14, 2020

Published: December 17, 2020

Copyright $\odot 2020$ by author(s) and Scientific Research Publishing Inc. This work is licensed under the Creative Commons Attribution International License (CC BY 4.0).

http://creativecommons.org/licenses/by/4.0/

\section{(c) (i) Open Access}

\begin{abstract}
Finite-dimensional integrable Hamiltonian system, obtained through the nonlinearization of the $3 \times 3$ spectral problem associated with the Boussinesq equation, is investigated. A generating function method starting from the Lax-Moser matrix is used to give an effective way to prove the involutivity of integrals. Finite-parameter solution of the Boussinesq equation is calculated based on the commutative system of ordinary differential equations with these integrals as Hamiltonians. The problem of the third order differential operator associated with the Boussinesq Neumann system put forward by $\mathrm{H}$. Flaschka in 1983 is solved.
\end{abstract}

Keywords

Boussinesq Equation, Conserved Integrals, Lax-Moser Matrix

\section{Introduction}

Boussinesq equation

$$
v_{t t}=-\frac{1}{3}\left(v_{x x}-4 v^{2}\right)_{x x}
$$

is one of the difficult soliton equations, which has been paid common attention in physical and mathematical fields [1]-[6]. In 1983, H. Flaschka put forward a problem of the third order differential operator associated with the Boussinesq Neumann system [7]. Some works focus on the decomposition and the structures of the Modified Boussinesq equation [8] [9] [10] [11]. The decomposition of the Boussinesq Neumann system has not been done thoroughly for a long time. A Neumann system of the Boussinesq equation associated with the third 
order differential operator is obtained in this paper, which is the extension of the famous KdV Neumann system associated with the second order differential operator. There are many methods to deal with the integrability and involutivity [12] [13] [14]. A generating function method starting from the Lax-Moser matrix [15]-[20] is used to give an effective way to prove the involutivity of integrals.

\section{The Generating Function of Integrals}

Let $R^{6 N}$ be the phase space. The canonical coordinates are

$$
q^{i}=\left(q_{1}^{i}, \cdots, q_{N}^{i}\right), \quad p^{i}=\left(p_{1}^{i}, \cdots, p_{N}^{i}\right), \quad i=1,2,3 .
$$

Write

$$
q=\left(q^{1}, q^{2}, q^{3}\right), \quad p=\left(p^{1}, p^{2}, p^{3}\right) ; \quad q_{k}=\left(q_{k}^{1}, q_{k}^{2}, q_{k}^{3}\right), \quad p_{k}=\left(p_{k}^{1}, p_{k}^{2}, p_{k}^{3}\right),
$$

for short. Take $A=\operatorname{diag}\left(\alpha_{1}, \cdots, \alpha_{N}\right)$, where $\alpha_{1}, \cdots, \alpha_{N}$ are distinct spectral constants. We denote:

$$
\langle X, Y\rangle \triangleq \sum_{k=1}^{N} X_{k} Y_{k}, \quad r^{i j}=\left\langle q^{i}, p^{j}\right\rangle, \quad A^{i j}=\left\langle A q^{i}, p^{j}\right\rangle,
$$

where $X$ and $Y$ are two $N$ dimensional vectors.

For any matrix $M=\left(M^{i j}\right)$, the element of its adjoint $M^{*}$ is $M_{i j}^{*}=(-1)^{i+j} M_{j i}$, where $M_{j i}$ is the cofactor of the element $M^{i j}$ in $M$.

In order to proof the Liouville integrability of the Hamiltonian system, consider the Lax-Moser matrix defined as:

$$
V_{\lambda}=V_{\lambda}(p, q)=\left(Q_{\lambda}^{i j}\right)_{3 \times 3}+l_{\lambda},
$$

where

$$
Q_{\lambda}^{i j}=Q_{\lambda}\left(q^{i}, p^{j}\right) \triangleq \sum_{k=1}^{N} \frac{q_{k}^{i} p_{k}^{j}}{\lambda-\alpha_{k}}, \quad l_{\lambda}=l_{\lambda}(p, q)=\left(\begin{array}{ccc}
0 & 0 & r^{32} \\
r^{32} & 0 & 2 r^{12}-r^{31} \\
0 & 0 & 0
\end{array}\right) .
$$

Let $L_{\lambda \xi}=\xi I_{3}+V_{\lambda}$. Then we have the generating function of integrals:

$$
F_{\lambda \xi}=\operatorname{det} L_{\lambda \xi}=\xi^{3}+F_{1}(\lambda) \xi^{2}+F_{2}(\lambda) \xi+F_{3}(\lambda),
$$

where

$$
\begin{aligned}
& F_{1}(\lambda)=Q_{\lambda}^{11}+Q_{\lambda}^{22}+Q_{\lambda}^{33}, \\
& F_{2}(\lambda)=\left|\begin{array}{cc}
Q_{\lambda}^{11} & Q_{\lambda}^{12} \\
Q_{\lambda}^{21}+r^{32} & Q_{\lambda}^{22}
\end{array}\right|+\left|\begin{array}{cc}
Q_{\lambda}^{22} & Q_{\lambda}^{23}+2 r^{12}-r^{31} \\
Q_{\lambda}^{32} & Q_{\lambda}^{33}
\end{array}\right|+\left|\begin{array}{cc}
Q_{\lambda}^{11} & Q_{\lambda}^{13}+r^{32} \\
Q_{\lambda}^{31} & Q_{\lambda}^{33}
\end{array}\right|, \\
& F_{3}(\lambda)=\left|\begin{array}{ccc}
Q_{\lambda}^{11} & Q_{\lambda}^{12} & Q_{\lambda}^{13}+r^{32} \\
Q_{\lambda}^{21}+r^{32} & Q_{\lambda}^{22} & Q_{\lambda}^{23}+2 r^{12}-r^{31} \\
Q_{\lambda}^{31} & Q_{\lambda}^{32} & Q_{\lambda}^{33}
\end{array}\right| .
\end{aligned}
$$

A series of polynomials $F_{j m}=F_{j m}(q, p)$, are defined as the coefficients of the power series expansions as $|\lambda|>\max \left(\left|\alpha_{1}\right|, \cdots,\left|\alpha_{N}\right|\right)$ : 


$$
F_{j}(\lambda)=\sum_{m=0}^{\infty} \lambda^{-m-1} F_{j m}, \quad j=1,2,3 .
$$

The first few are expressed as $|\lambda|>\max \left(\left|\alpha_{1}\right|, \cdots,\left|\alpha_{N}\right|\right)$ :

$$
\begin{aligned}
F_{10}= & r^{11}+r^{22}+r^{33}, \quad F_{20}=-3 r^{12} r^{32}, \quad F_{30}=\left(r^{32}\right)^{3}, \\
F_{21}= & -r^{32}\left(A^{12}+A^{31}\right)-\left(2 r^{12}-r^{31}\right) A^{32}+\sum_{1 \leq i<j \leq 3}\left(r^{i i} r^{i j}-r^{i j} r^{j i}\right), \\
F_{31}= & \left(r^{32}\right)^{2} A^{32}+r^{32}\left(r^{21} r^{32}-r^{22} r^{31}+r^{13} r^{32}-r^{12} r^{33}\right) \\
& -\left(2 r^{12}-r^{31}\right)\left(r^{11} r^{32}-r^{12} r^{31}\right) .
\end{aligned}
$$

By comparing the coefficients of the same power of $\lambda$, general explicit formulas are obtained:

$$
\begin{aligned}
& F_{1 m}=\left\langle A^{m} q^{1}, p^{1}\right\rangle+\left\langle A^{m} q^{2}, p^{2}\right\rangle+\left\langle A^{m} q^{3}, p^{3}\right\rangle, \quad m \geq 1 ; \\
& F_{2 m}=-r^{32}\left(\left\langle A^{m} q^{1}, p^{2}\right\rangle+\left\langle A^{m} q^{3}, p^{1}\right\rangle\right)-\left(2 r^{12}-r^{31}\right)\left\langle A^{m} q^{3}, p^{2}\right\rangle \\
& +\sum_{k+l=m-1} \sum_{\substack{k \leq l<j<j \leq 3 \\
k, l \geq 0}}\left|\begin{array}{ll}
\left\langle A^{k} q^{i}, p^{i}\right\rangle & \left\langle A^{l} q^{i}, p^{j}\right\rangle \\
\left\langle A^{k} q^{j}, p^{i}\right\rangle & \left\langle A^{l} q^{j}, p^{j}\right\rangle
\end{array}\right|, \quad m \geq 2 ; \\
& F_{3 m}=\left(r^{32}\right)^{2}\left\langle A^{m} q^{3}, p^{2}\right\rangle \\
& +r^{32} \sum_{k+l=m-1}\left(\left|\begin{array}{ll}
\left\langle A^{k} q^{2}, p^{1}\right\rangle & \left\langle A^{l} q^{2}, p^{2}\right\rangle \\
\left\langle A^{k} q^{3}, p^{1}\right\rangle & \left\langle A^{l} q^{3}, p^{2}\right\rangle
\end{array}\right|-\left|\begin{array}{ll}
\left\langle A^{k} q^{1}, p^{2}\right\rangle & \left\langle A^{l} q^{1}, p^{3}\right\rangle \\
\left\langle A^{k} q^{3}, p^{2}\right\rangle & \left\langle A^{l} q^{3}, p^{3}\right\rangle
\end{array}\right|\right) \\
& -\left(2 r^{12}-r^{31}\right) \sum_{\substack{k+l=m-1 \\
k, l \geq 0}}\left|\begin{array}{ll}
\left\langle A^{k} q^{1}, p^{1}\right\rangle & \left\langle A^{l} q^{1}, p^{2}\right\rangle \\
\left\langle A^{k} q^{3}, p^{1}\right\rangle & \left\langle A^{l} q^{3}, p^{2}\right\rangle
\end{array}\right| \\
& +\sum_{j+k+l=m-2}\left|\begin{array}{lll}
\left\langle A^{j} q^{1}, p^{1}\right\rangle & \left\langle A^{k} q^{1}, p^{2}\right\rangle & \left\langle A^{l} q^{1}, p^{3}\right\rangle \\
\left\langle A^{j} q^{2}, p^{1}\right\rangle & \left\langle A^{k} q^{2}, p^{2}\right\rangle & \left\langle A^{l} q^{2}, p^{3}\right\rangle \\
\left\langle A^{j} q^{3}, p^{1}\right\rangle & \left\langle A^{k} q^{3}, p^{2}\right\rangle & \left\langle A^{l} q^{3}, p^{3}\right\rangle
\end{array}\right|, \quad m \geq 2 .
\end{aligned}
$$

Expand $F_{j}(\lambda)$ in non-negative power of $\lambda$ :

$$
F_{j}(\lambda)=\sum_{m=1}^{\infty} \lambda^{m-1} F_{j,-m}, \quad j=1,2,3
$$

as $|\lambda|<\max \left(\left|\alpha_{1}\right|, \cdots,\left|\alpha_{N}\right|\right)$. By comparing the coefficients of the same power of $\lambda$, we have:

$$
\begin{aligned}
F_{1,-m}= & -\left(\left\langle A^{-m} q^{1}, p^{1}\right\rangle+\left\langle A^{-m} q^{2}, p^{2}\right\rangle+\left\langle A^{-m} q^{3}, p^{3}\right\rangle\right), \quad m \geq 1 ; \\
F_{2,-m}= & r^{32}\left(\left\langle A^{-m} q^{1}, p^{2}\right\rangle+\left\langle A^{-m} q^{3}, p^{1}\right\rangle\right) \\
& +\left(2 r^{12}-r^{31}\right)\left\langle A^{-m} q^{3}, p^{2}\right\rangle \\
& +\sum_{\substack{k+l=m+1 \\
k, l \geq 1}} \sum_{\substack{1 \leq i<j \leq 3 \\
j}}\left|\left\langle A^{-k} q^{i}, p^{i}\right\rangle\left\langle A^{-k} q^{j}, p^{i}\right\rangle\left\langle A^{-l} q^{i}, p^{j}\right\rangle\right|, \quad m \geq 1 ;
\end{aligned}
$$




$$
\begin{aligned}
& F_{3,-m}=-\left(r^{32}\right)^{2}\left\langle A^{m} q^{3}, p^{2}\right\rangle \\
& \left.+r^{32} \sum_{\substack{k+l=m+1 \\
k, l \geq 1}}\left(\left|\begin{array}{ll}
\left\langle A^{-k} q^{2}, p^{1}\right\rangle & \left\langle A^{-l} q^{2}, p^{2}\right\rangle \\
\left\langle A^{-k} q^{3}, p^{1}\right\rangle & \left\langle A^{-l} q^{3}, p^{2}\right\rangle
\end{array}\right|-\mid \begin{array}{ll}
\left\langle A^{-k} q^{1}, p^{2}\right\rangle & \left\langle A^{-l} q^{1}, p^{3}\right\rangle \\
\left\langle A^{-k} q^{3}, p^{2}\right\rangle & \left\langle A^{-l} q^{3}, p^{3}\right\rangle
\end{array}\right)\right) \\
& -\left(2 r^{12}-r^{31}\right) \sum_{\substack{k+l=m+1 \\
k, l \geq 1}}\left|\begin{array}{ll}
\left\langle A^{-k} q^{1}, p^{1}\right\rangle & \left\langle A^{-l} q^{1}, p^{2}\right\rangle \\
\left\langle A^{-k} q^{3}, p^{1}\right\rangle & \left\langle A^{-l} q^{3}, p^{2}\right\rangle
\end{array}\right| \\
& -\sum_{j+k+l=m+2}\left|\begin{array}{lll}
\left\langle A^{-j} q^{1}, p^{1}\right\rangle & \left\langle A^{-k} q^{1}, p^{2}\right\rangle & \left\langle A^{-l} q^{1}, p^{3}\right\rangle \\
\left\langle A^{-j} q^{2}, p^{1}\right\rangle & \left\langle A^{-k} q^{2}, p^{2}\right\rangle & \left\langle A^{-l} q^{2}, p^{3}\right\rangle \\
\left\langle A^{-j} q^{3}, p^{1}\right\rangle & \left\langle A^{-k} q^{3}, p^{2}\right\rangle & \left\langle A^{-l} q^{3}, p^{3}\right\rangle
\end{array}\right|, \quad m \geq 1 .
\end{aligned}
$$

\section{The Involutivity of Integrals}

The involutivity is critical to the integrability of the Hamiltonian system, which is defined as the Poisson bracket of two conserved integrals being zero. A direct calculation gives:

Proposition 1. The Hamiltonian system for the $F_{\lambda \xi}$-flow is expressed as:

$$
\frac{\mathrm{d} q_{k}}{\mathrm{~d} t_{\lambda \xi}}=W_{\lambda \xi \mu} q_{k}, \quad \frac{\mathrm{d} p_{k}}{\mathrm{~d} t_{\lambda \xi}}=-W_{\lambda \xi \mu}^{\mathrm{T}} p_{k},
$$

where

$$
W_{\lambda \xi \mu}=\frac{1}{\lambda-\alpha_{k}} L_{\lambda \xi}^{*}+L_{0 \lambda \xi}, L_{0 \lambda \xi}=\left(\begin{array}{ccc}
0 & 0 & L_{23} \\
-2 L_{23} & 0 & L_{13}-L_{21} \\
0 & 0 & 0
\end{array}\right) .
$$

Proof. From the Equation (3) and the property of Hamiltonian system, we calculate the partial derivatives of $q_{k}$ and $p_{k}$ with respect to $t_{\lambda \xi}$, then the results are obtained.

Lemma 2. $\varepsilon_{k}=q_{k} p_{k}^{\mathrm{T}}$ satisfies the Lax equation along the $t_{\lambda \xi}$-flow:

$$
\frac{\mathrm{d} \varepsilon_{k}}{\mathrm{~d} t_{\lambda \xi}}=\left[W_{\lambda \xi \mu}, \varepsilon_{k}\right]=\frac{1}{\lambda-\alpha_{k}}\left[L_{\lambda \xi}^{*}, \varepsilon_{k}\right] .
$$

Proof.

$$
\dot{\varepsilon}_{k}=\dot{q}_{k} p_{k}^{\mathrm{T}}+q_{k} \dot{p}_{k}^{\mathrm{T}}=\left(W q_{k}\right) p_{k}^{\mathrm{T}}+q_{k}\left(-W^{\mathrm{T}} q_{k}\right)^{\mathrm{T}}=W \varepsilon_{k}-\varepsilon_{k} W=\left[W, \varepsilon_{k}\right] \text {. }
$$

Proposition 3. $L_{\mu \eta}$ satisfies the Lax equation along the $t_{\lambda \xi}$-flow:

$$
\frac{\mathrm{d} L_{\mu \eta}}{\mathrm{d} t_{\lambda \xi}}=\left[W_{\lambda \xi \mu}, L_{\mu \eta}\right] \text {. }
$$

Proof. $Q_{\mu}$ can be expressed by $\varepsilon_{k}$ as:

$$
Q_{\mu}=\sum_{k=1}^{N} \frac{\varepsilon_{k}}{\mu-\alpha_{k}} .
$$

Resorting to Lemma 2, we have:

$$
\frac{\mathrm{d} Q_{\mu}}{\mathrm{d} t_{\lambda \xi}}=\left[W_{\lambda \xi \mu}, L_{\mu \eta}\right]-\left[L_{0 \lambda \xi}, l_{\mu}\right],
$$




$$
\frac{\mathrm{d} L_{\mu \eta}}{\mathrm{d} t_{\lambda \xi}}-\left[W_{\lambda \xi \mu}, L_{\mu \eta}\right]=\frac{\mathrm{d} l_{\mu}}{\mathrm{d} t_{\lambda \xi}}-\left[L_{0 \lambda \xi}, l_{\mu}\right]=0 .
$$

Lemma 4. The two determinants are true as $a, b, c, d=1,2,3$ :

$$
\begin{aligned}
& \left|\begin{array}{ll}
Q_{\lambda}\left(q^{a}, p^{b}\right) & Q_{\lambda}\left(q^{a}, p^{d}\right) \\
Q_{\lambda}\left(q^{c}, p^{b}\right) & Q_{\lambda}\left(q^{c}, p^{d}\right)
\end{array}\right|=\frac{1}{2} \sum_{k=1}^{N} \sum_{l=1}^{N} \frac{1}{\left(\lambda-\alpha_{k}\right)\left(\lambda-\alpha_{l}\right)}\left|\begin{array}{ll}
q_{k}^{a} & q_{l}^{a} \\
q_{k}^{c} & q_{l}^{c}
\end{array}\right|\left|\begin{array}{ll}
p_{k}^{b} & p_{k}^{d} \\
p_{l}^{b} & p_{l}^{d}
\end{array}\right| . \\
& \operatorname{det}\left(Q_{\lambda}\left(q^{i}, p^{j}\right)\right)_{3 \times 3} \\
& =\frac{1}{6} \sum_{j=1}^{N} \sum_{k=1}^{N} \sum_{l=1}^{N} \frac{1}{\left(\lambda-\alpha_{j}\right)\left(\lambda-\alpha_{k}\right)\left(\lambda-\alpha_{l}\right)}\left|\begin{array}{ccc|ccc}
q_{j}^{1} & q_{k}^{1} & q_{l}^{1} \\
q_{j}^{2} & q_{k}^{2} & q_{l}^{2} \\
q_{j}^{3} & q_{k}^{3} & q_{l}^{3}
\end{array}\right| \begin{array}{ccc}
p_{j}^{1} & p_{k}^{2} & p_{l}^{3} \\
p_{j}^{1} & p_{k}^{2} & p_{l}^{3} \\
p_{j}^{1} & p_{k}^{2} & p_{l}^{3}
\end{array} \mid .
\end{aligned}
$$

From (6) and (7), we have:

$$
\begin{gathered}
F_{1}(\lambda)=F_{10} \frac{\beta_{1}(\lambda)}{\alpha(\lambda)}=\left(r^{11}+r^{22}+r^{33}\right) \frac{\beta_{1}(\lambda)}{\alpha(\lambda)}=\sum_{k=1}^{N} \frac{E_{1 k}}{\lambda-\alpha_{k}}, \\
F_{2}(\lambda)=F_{20} \frac{\beta_{2}(\lambda)}{\alpha(\lambda)}=-3 r^{12} r^{32} \frac{\beta_{2}(\lambda)}{\alpha(\lambda)}=\sum_{k=1}^{N} \frac{E_{2 k}}{\lambda-\alpha_{k}}, \\
F_{3}(\lambda)=F_{30} \frac{\beta_{3}(\lambda)}{\alpha(\lambda)}=\left(r^{32}\right)^{3} \frac{\beta_{3}(\lambda)}{\alpha(\lambda)}=\sum_{k=1}^{N} \frac{E_{3 k}}{\lambda-\alpha_{k}},
\end{gathered}
$$

where $\alpha(\lambda)=\prod_{k=1}^{N}\left(\lambda-\alpha_{k}\right)$, and $\beta_{1}(\lambda), \beta_{2}(\lambda), \beta_{3}(\lambda)$ are polynomials of degree $N-1$. From (8)-(10), another group of conserved integrals $E_{1 k}, E_{2 k}, E_{3 k}$ is obtained:

$$
\begin{aligned}
& E_{1 k}=\sum_{i=1}^{3} q_{k}^{i} p_{k}^{i}, \quad 1 \leq k \leq N ; \\
& E_{2 k}=-r^{32}\left(q_{k}^{1} p_{k}^{2}+q_{k}^{3} p_{k}^{1}\right)-\left(2 r^{12}-r^{31}\right) q_{k}^{3} p_{k}^{2}
\end{aligned}
$$

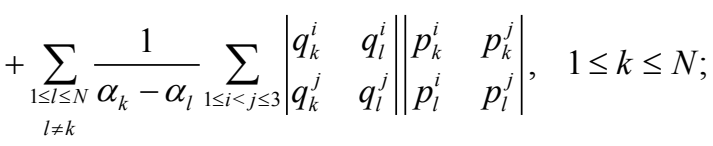

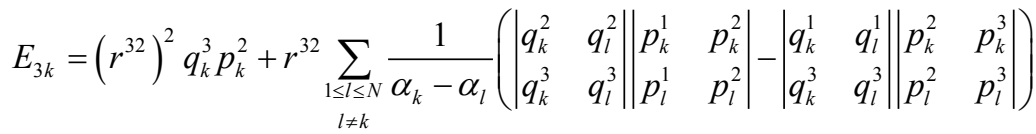

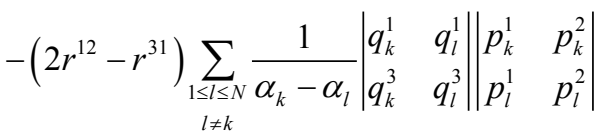

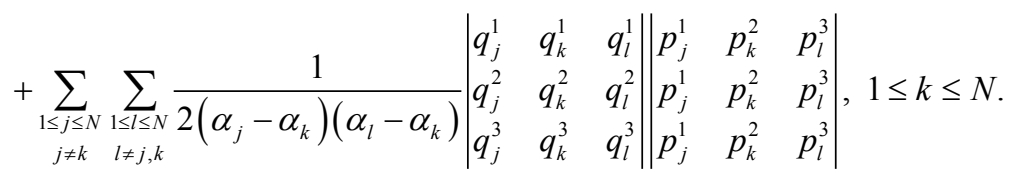

From the definition of involutivity of two conserved integrals and direct calcultion, we have:

Proposition 5. The integrals

$$
\left\{F_{1 m}, F_{2 m}, F_{3 m}, m \in Z ; E_{1 k}, E_{2 k}, E_{3 k}, 1 \leq k \leq N\right\}
$$


are involutive in pairs:

$$
\begin{gathered}
\left(F_{i m}, F_{j n}\right)=0, \quad \forall i, j=1,2,3 ; \quad \forall m, n=0,1,2, \cdots \\
\left(F_{i,-m}, F_{j,-n}\right)=0, \quad \forall i, j=1,2,3 ; \quad \forall m, n=, 1,2, \cdots \\
\left(F_{i m}, F_{j,-n}\right)=0, \quad \forall i, j=1,2,3 ; \quad \forall m=0,1,2, \cdots ; n=1,2, \cdots \\
\left(E_{i k}, E_{j l}\right)=0, \quad \forall i, j=1,2,3 ; \quad \forall 1 \leq k, l \leq N . \\
\left(F_{i m}, E_{j l}\right)=0, \quad \forall i, j=1,2,3 ; \quad \forall m \in Z ; 1 \leq l \leq N .
\end{gathered}
$$

\section{Hamiltonian Systems}

By direct calculations, the canonical equations of the Hamiltonian systems $\left(F_{31}\right)$ and $\left(F_{21}\right)$ can be expressed as:

$$
\begin{aligned}
& q_{x}=\frac{\partial F_{31}}{\partial p}=U q, \quad p_{x}=-\frac{\partial F_{31}}{\partial q}=-U^{\mathrm{T}} p ; \\
& q_{t}=\frac{\partial F_{21}}{\partial p}=V q, \quad p_{t}=-\frac{\partial F_{21}}{\partial q}=-V^{\mathrm{T}} p ;
\end{aligned}
$$

where $U, V$ are $3 N \times 3 N$ matrices:

$$
\begin{gathered}
U=\left(\begin{array}{ccc}
r^{31} & 1 & r^{11}-r^{22} \\
-\left[2 r^{11}+r^{33}+\left(r^{31}\right)^{2}\right] & -r^{31} & A+2 A^{32}+2 r^{13}+2 r^{21}+\left(r^{11}-r^{22}\right) r^{31} \\
1 & 0 & 0
\end{array}\right) ; \\
V=\left(\begin{array}{ccc}
r^{22}+r^{33} & 0 & -A+A^{32}-r^{13} \\
-\left(A+2 A^{32}+r^{21}\right) & r^{11}+r^{33} & r^{31} A-A^{12}-A^{31}-r^{23} \\
-r^{31} & -1 & r^{11}+r^{22}
\end{array}\right) .
\end{gathered}
$$

Proposition 6. Let $q(x, t), p(x, t)$ be compatible solution of the $\left(F_{31}\right)$ and $\left(F_{21}\right)$ flow on the level set $\left\{(p, q) \in \mathbb{R}^{6 N}: r^{32}=1, r^{12}=0\right\}$. Then the Boussinesq Equation (1) has a finite-parameter solution $(u, v)=B(q, p)$ given as:

$$
u=-\frac{3}{2} A^{32}+\frac{1}{2} F_{31}, \quad v=\frac{3}{2} r^{11}-\frac{1}{2} F_{10} .
$$

Proof. A direct calculation gives:

$$
\begin{aligned}
r_{x}^{11} & =-r^{13}+r^{21}+\left(r^{11}-r^{22}\right) r^{31}, \\
r_{x}^{22} & =3 A^{32}+2 r^{13}+r^{21}+\left(r^{11}-r^{22}\right) r^{31}, \\
r_{x}^{33} & =-3 A^{32}-r^{13}-2 r^{21}-2\left(r^{11}-r^{22}\right) r^{31}, \\
r_{x}^{13} & =-A^{12}+r^{23}+r^{13} r^{31}+\left(r^{11}-r^{22}\right)\left(r^{33}-r^{11}\right), \\
r_{x}^{21} & =A^{31}+2 r^{31} A^{32}-r^{23}+2 r^{13} r^{31}-\left(r^{11}-r^{22}\right)\left(2 r^{11}+r^{33}\right), \\
r_{x}^{23}= & A^{33}-A^{22}+2\left(r^{33}-r^{22}\right) A^{32}-r^{23} r^{31}+\left(-2 r^{11}-2 r^{22}+r^{33}\right) r^{13}, \\
& +\left(-r^{11}-r^{22}+2 r^{33}\right) r^{21}-r^{13}\left(r^{31}\right)^{2}+\left(r^{11}-r^{22}\right)\left(r^{33}-r^{22}\right) r^{31},
\end{aligned}
$$




$$
\begin{aligned}
& r_{x}^{31}=3 r^{11}, \quad A_{x}^{12}=-A^{11}+A^{22}+2 r^{31} A^{21}+\left(r^{11}-r^{22}\right) A^{32}, \\
& A_{x}^{31}=A^{11}-A^{33}-r^{31} A^{31}+\left[2 r^{11}+r^{33}+\left(r^{31}\right)^{2}\right] A^{32}, \\
& A_{x}^{32}=A^{12}-A^{31}+r^{31} A^{32} .
\end{aligned}
$$

So from the above calculations and (16), we have:

$$
v_{t}=-u_{x}, \quad u_{t}=\frac{1}{3}\left(v_{x x}-4 v^{2}\right)_{x},
$$

and

$$
v_{t t}=\left(-u_{x}\right)_{t}=-\left(u_{t}\right)_{x}=-\frac{1}{3}\left(v_{x x}-4 v^{2}\right)_{x x}
$$

which is exactly the "good" Boussinesq Equation (1).

\section{Conclusion}

The third order differential operator associated with the Boussinesq Neumann system is a critical point in researching problems of integrable system. In this paper, we obtain a Neumann system of the Boussinesq equation associated with the third order differential operator, which is the extension of the famous KdV Neumann system associated with the second order differential operator. By means of generating function from the Lax-Moser matrix, we prove the involutivity of integrals successfully. Meanwhile, a finite-parameter solution to the Boussinesq equation is obtained naturally.

\section{Acknowledgements}

This work is supported by the Foundation (Grant No. 11601123 and 202010463050).

\section{Conflicts of Interest}

The authors declare no conflicts of interest regarding the publication of this paper.

\section{References}

[1] Tu, G.Z. (1981) The Backlund Transformation and Conserved Law for the Boussinesq Equation. Acta Mathematicae Applicatae Sinica, 1, 63-68.

[2] Deift, P., Tomei, C. and Trubowitz, E. (1982) Inverse Scattering and the Boussinesq Equation. Communications on Pure and Applied Mathematics, 35, 567-628. https://doi.org/10.1002/cpa.3160350502

[3] Ortega, T. and Sanz-Serna, J.M. (1990) Nonlinear Stability and Convergence of Finite-Difference Methods for the "Good" Boussinesq Equation. Numerische Mathematik, 58, 215-229. https://doi.org/10.1007/BF01385620

[4] Schaffer, H.A., Madsen, P.A. and Deigaard, R. (1993) A Boussinesq Model for Waves Breaking in Shallow Water. Coastal Engineering, 20, 185-202. https://doi.org/10.1016/0378-3839(93)90001-O

[5] Hu, X.B., Li, Y. and Liu, Q.M. (1993) Nonlinear Superposition Formula of the Boussinesq Hierarchy. Acta Mathematicae Applicatae Sinica, 1, 17-27. 
[6] Ma, W.X. (1993) On the Complete Integrability of Nonlinearized Lax Systems for the Classical Boussinesq Hierarchy. Acta Mathematicae Applicatae Sinica, 1, 92-96.

[7] Flaschka, H. (1983) Relations between Infinite-Dimensional and Finite-Dimensional Isospectral Equations. Proceedings of RIMS Symposium on Nonlinear Integrable Systems, Kyoto, Japan, World Sci. Pub., Singapore, 219-239.

[8] Zeng, Y.B. (1995) The Variable Separation of the Modified Boussinesq. Annual Mathematics, 1, 50-59.

[9] Conte, R., Musette, M. and Pickering, A. (1995) The Two-Singular Manifold Method: II. Classical Boussinesq System. Journal of Physics A: Mathematical and General, 28, 179-187. https://doi.org/10.1088/0305-4470/28/1/020

[10] Dai, H.H. and Geng, X.G. (2003) Finite-Dimensional Integrable Systems through the Decomposition of a Modified Boussinesq Equation. Physics Letters A, 317, 389-400. https://doi.org/10.1016/j.physleta.2003.08.049

[11] Dougalis, V.A., Durn, A., Lpez-Marcos, M.A. and Mitsotakis, D.E. (2007) A Numerical Study of the Stability of Solitary Waves of the Bona-Smith Family of Boussinesq Systems. Journal of Nonlinear Science, 17, 569-607. https://doi.org/10.1007/s00332-007-9004-8

[12] Lax, P.D. (1968) Integrals of Nonlinear Equations of Evolution and Solitary Waves. Communications on Pure and Applied Mathematics, 21, 467-490. https://doi.org/10.1002/cpa.3160210503

[13] Ma, W.X. (1990) The Confocal Involutive Systems and the Integrability of the Nonlinearized Lax Systems of AKNS Hierarchy. In: Gu, C., Li, Y., Tu, G. and Zeng, Y., Eds., Nonlinear Physics, Research Reports in Physics, Springer-Verlag, Berlin, 79-84. https://doi.org/10.1007/978-3-642-84148-4_10

[14] Wang, X., Cao, J.L. and Chen, Y. (2015) Higher-Order Rogue Wave Solutions of the Three-Wave Resonant Interaction Equation via the Generalized Darboux Transformation. Physica Scripta, 90, 10. https://doi.org/10.1088/0031-8949/90/10/105201

[15] Cao, C.W. (1990) Nonlinearization of the Lax System for AKNS Hierarchy. Science China $A, 33,528-536$.

[16] Cao, C.W. and Cao, J.L. (2006) On an Integrable (2+1) Dimensional Korteweg-de Vries Equation with a Discrete Variable. Il Nuovo Cimento B, 121, 675-687.

[17] Cao, C.W. and Cao, J.L. (2007) An Integrable (2+1)-Dimensional Toda Equation with Two Discrete Variables. Physics Letters A, 365, 301-308. https://doi.org/10.1016/j.physleta.2007.01.028

[18] Cao, J.L. (2007) Finite Parameter Solutions to the Manakov and the Derivative Manakov Equations. Chinese Quarterly Journal of Mathematics, 22, 236-244.

[19] Cao, J.L., Zhang, H. and Jiao, W.T. (2008) Coupled KdV Equations and Their Explicit Solutions Through Two-Dimensional Hamiltonian System with a Quartic Potential. Communications in Theoretical Physics, 49, 1379-1382. https://doi.org/10.1088/0253-6102/49/6/04

[20] Cao, J.L. and Shi, W. (2013) A New Integrable Generalized Neumann System Related to Soliton Hierarchy. Chaos, Solitons and Fractals, 47, 13-17. https://doi.org/10.1016/j.chaos.2012.11.005 